Pacific Journal of Mathematic 


\title{
EQUATIONAL COMPACTNESS AND COMPACT TOPOLOGIES IN RINGS SATISFYING A.C.C.
}

\author{
David K. Haley
}

In a 1962 paper $\mathrm{S}$. Warner posed the following question: for which classes of rings does each compact topological ring therein possess a unique compact topology? There it is shown that the class of (unital) noetherian rings has this property, the topology in question being the Jacobson radical topology; in another paper he showed that the class of semisimple rings enjoys the same property, this latter result drawing heavily on I. Kaplansky's structure theorem for compact semisimple rings. In particular a compact topological ring with no nonzero topological nilpotents is in possession of the only compact topology it can carry. In this report we investigate equational compactness in the class of arbitrary (associative) rings satisfying the ascending chain condition on left ideals (A.C.C.). Now the theory of equational compactness has been probed in various classes of (universal) algebras and in many cases this algebraic property characterizes the topological-algebraic property that an algebra be a retract of a compact topological algebra (the "Mycielski Problem"). We showed that in the class of commutative noetherian rings equational compactness is equivalent to topological compactness, so a strong motivating factor prompting a further investigation was the suspicion that the sharpened result obtained in the commutative noetherian case could be substantially generalized - yielding, first, an answer to the Mycielski Problem in this larger class of rings and, secondly, providing additional algebraic footholds for a further assault on Warner's question. Here positive answers to both questions are obtained for the class of rings with A.C.C.

0. Background. In this section relevant concepts are sketched and those results listed which are needed in the sequel but which are already known.

A universal algebra $\mathfrak{U}$ is equationally compact (J. Mycielski [11]) if every system $\Sigma$ of polynomial equations with constants in $\mathfrak{A}$ is simultaneously solvable in $\mathfrak{U}$ provided every finite subset of $\Sigma$ is. (See G. Grätzer [3] for universal algebraic and model-theoretic concepts.) $\mathfrak{U}$ is positively compact if the same relative solvability condition holds for systems of positive formulas with constants in $\mathfrak{A}$. (A positive formula with constants in $\mathfrak{A}$ is a formula of the first order predicate calculus built up from 
polynomial equations with constants in $\mathfrak{U}$ by applying only the logical connectives $\wedge, \vee$ and the quantifiers $\exists, \forall$.) The following modeltheoretic result will be of use in the sequel.

Proposition 0.1 (B. Weglorz [18]). A universal algebra is equationally compact if and only if it is positively compact.

A compact topological (universal) algebra $\mathfrak{A}$ is an algebra endowed with a compact (Hausdorff) topology compatible with its structure. It is an easy consequence of the Tychonoff Theorem that every compact topological algebra is equationally compact (see $\mathrm{J}$. Mycielski [11]), as is any retract of an equationally compact algebra. Thus the "Mycielski Problem" mentioned at the outset reads: In what classes are the equationally compact algebras always retracts of compact topological algebras? W. Taylor proved in [12] that there are equationally compact algebras which are not retracts of compact topological algebras, and indeed has constructed a semigroup with this property (see [13]). Nonetheless, numerous classes of algebras are known supporting the positive side: Boolean algebras, semilattices, mono-unary algebras, unital modules, artinian rings, and varieties generated by quasi-primal algebras (see [18], [2], [19], [15], [5], resp. [14]); in this report we add rings satisfying A.C.C. to the list.

Now a ring is a structural enrichment of an abelian group; hence a ring $R$ is equationally compact only if $R^{+}$, the abelian group underlying it, is equationally compact. Equationally compact abelian groups, however, have a nice description. This is

DEFINITION 0.2 (I. Kaplansky [8]). An abelian group $G$ is algebraically compact if

$$
G \cong D \oplus \Pi\left(G_{p} ; p \in \mathbf{P}\right)
$$

where $D$ is divisible, $\mathbf{P}$ denotes the set of prime natural numbers, and for each $p \in \mathbf{P}, G_{p}$ is a module over the $p$-adic integers $\mathbf{Z}_{p}^{*}$, the $p$-adic topology on $G_{p}$ is Hausdorff, and $G_{p}$ is complete in it.

J. Koś proved in [9] that equational compactness in abelian groups is characterized by algebraic compactness, and we shall exploit this property in equationally compact rings. To this end, the following internal description of the factors of an algebraically compact group will prove useful.

Proposition 0.3 (I. Kaplansky [8]). If $G=D \oplus \Pi\left(G_{p} ; p \in \mathbf{P}\right)$ is an algebraically compact group, then - viewing the factors as subgroups 
in the canonical fashion $-D$ is the largest divisible subgroup of $G$, and for each prime number $p, G_{p}$ is the largest subgroup of $G$ divisible by all powers of all primes excepting $p$.

Let $R$ be an (associative) ring. We say $\mathscr{T}$ is a topology for $R$ if $(R, \mathscr{T})$ is a topological ring. $\quad R$ is a compact ring, provided there exists a compact topology for $R$ (where compact always means quasi-compact and Hausdorff). $\quad R$ is noetherian if $R$ is a unital ring and satisfies A.C.C. The characteristic of $R, \chi(R)$, is defined to be the torsion bound of $R^{+}$if $R^{+}$is a bounded torsion group, and zero otherwise. $J(R)$ denotes the Jacobson radical of $R . \quad R$ is semisimple if $J(R)$ is zero. For general information on rings, topological rings, and ideal topologies consult [1], [6] and [20].

Proposition 0.4 (S. Warner [17]). Let $(R, \mathscr{T})$ be a topological noetherian ring. Then $(R, \mathscr{T})$ is a compact topological ring if and only if $\mathscr{T}$ is the $J(R)$-topology, $R$ is complete in it, $\cap\left(J(R)^{n} ; n \in \mathbf{N}\right)=(0)$, and $R / J(R)$ is finite.

Suppose that $A$ and $R$ are rings such that $A$ is unital and $R$ is an $A$-algebra which is unital as a (left) $A$-module. Denote by $A * R$ the ring obtained by the standard "adjunction of an identity" construction. That is, the carrier set is $A \times R$, addition is componentwise, and multiplication is given by $(a, r) \cdot(b, s)=(a \cdot b, a \cdot s+b \cdot r+r \cdot s)$. We identify $A$ and $R$ with their images under the canonical (ring) embeddings into $A * R$. Note that $A * R$ has an identity, $R$ is an ideal of $A * R$, and $A * R$ satisfies A.C.C. as soon as both $A$ and $R$ do. Proofs of the following results are in [5].

Proposition 0.5. Let $R$ be an equationally compact ring of characteristic $n>0$. Then $Z_{n} * R$ is equationally compact. $\left(Z_{n}\right.$ denotes the ring of integers modulo $n$.)

Proposition 0.6. Let $R$ be an equationally compact ring and $D$ a divisible subgroup of $R^{+}$. Then $R \cdot D=D \cdot R=\{0\}$.

Proposition 0.7. Every equationally compact semisimple noetherian ring is finite.

1. Preliminary results. It is most advantageous when equational compactness is preserved in passing from an algebra to a homomorphic image or to a subalgebra. This is not generally the case, but the definition following gives a criterion under which it is true in rings. A restricted form of this criterion appeared in [5], but in the present discussion a slightly broader concept is require $\dot{d}$. 
Definition 1.1. Let $R$ be a ring and $\mathfrak{A}$ a left ideal of $R$. $\quad \mathfrak{A}$ is positive in $R$ if there is a variable $x$ and a set $\Pi$ of positive formulas with constants in $R$, say $\Pi=\left\{\varphi_{1}(x) ; i \in I\right\}$, such that

(a) $x$ is the only variable occurring free in each $\varphi_{1}(x)$, and

(b) the solution set of $\Pi$ in $R$ (i.e., the set of substitutes from $R$ for the free occurrences of $x$ in $\varphi_{l}(x)$ making each $\varphi_{i}(x)$ true in $\left.R\right)$ is precisely $\mathfrak{A}$.

Given the above situation, we say that $\mathfrak{A}$ is expressible in $R$ by the formulas $\Pi$.

EXAMPLE 1.2. Let $R$ be a ring which either has an identity or is such that $R^{+}$is a torsion group. Let $\mathfrak{A}$ be a finitely generated left ideal of $R$ generated, say, by $a_{1}, \cdots, a_{n}$. If $R$ is unital, set $k=1$, and if $R^{+}$is torsion, let $k$ be a natural number at least as large as the order of each $a_{i}$, $i=1, \cdots, n$. Then $\mathfrak{A}$ is expressed in $R$ (in both cases) by the single formula

$$
\varphi(x)=\left(\exists x_{1}\right) \cdots\left(\exists x_{n}\right) \underset{\substack{0 \leq m_{1}<k \\ 1 \leq 1 \leq n}}{\vee}\left(x=x_{1} a_{1}+\cdots+x_{n} a_{n}+m_{1} a_{1}+\cdots+m_{n} a_{n}\right)
$$

Hence $\mathfrak{A}$ is positive in $R$.

ExAmPLE 1.3. Let $R$ be a ring. The Jacobson radical $J(R)$ is the largest left-quasi-regular left ideal in $R$, that is, $J(R)$ consists precisely of those elements of $R$ which generate principal left-quasi-regular left ideals. Thus $J(R)$ is expressible in $R$ by the set of formulas

$$
\Pi=\left\{\varphi_{s, z}(x) ; s \in R, z \in \mathbf{Z}\right\}
$$

with

$$
\varphi_{s, z}(x)=(\exists y)((s x+z x)+y+y(s x+z x)=0) .
$$

Hence $J(R)$ is positive in $R$.

Suppose $\mathfrak{A}$ is positive in the ring $R$ - expressible, say, by the set of formulas $\Pi$. Then in effect this just says that the unary relational predicate " $x \in \mathfrak{P}$ " is characterized by the set $\Pi$ of positive formulas in the sense that an element $r$ in $R$ is a solution of $x \in \mathfrak{A}$ (i.e., $r \in \mathfrak{U}$ ) if and only if $r$ is a solution of $\Pi$.

PROPOSITION 1.4. Let $R$ be an equationally compact ring, let $\mathfrak{A}_{j}$, $j \in J$, be a family of positive left ideals of $R$, and for each $j \in J$ let $x$, be a variable. Let $\Sigma$ be a set of polynomial equations with constants in R. Then 


$$
\Omega=\Sigma \cup\left\{x, \in \mathfrak{A}_{J} ; j \in J\right\}
$$

is solvable in $R$ provided it is finitely solvable in $R$.

Proof. Let $\mathfrak{A}$, be expressible by the set of positive formulas $\Pi_{j}$, $j \in J$. If $\Omega$ is finitely solvable, then so is the set of positive formulas

$$
\Sigma \cup \cup(\Pi, ; j \in J)
$$

which is then solvable by Proposition 0.1 . But then obviously $\Omega$ is too.

PROPOSITION 1.5. Let $R$ be an equationally compact ring and let $\mathfrak{A}$ and $\mathfrak{B}$ be positive ideals (resp. left ideals) with $\mathfrak{A} \supseteq \mathfrak{B}$. Then $\mathfrak{U} / \mathfrak{B}$ is an equationally compact ring (resp. $R$-module).

Proof. Suppose $\mathfrak{U}$ and $\mathfrak{B}$ are ideals and let $\Sigma$ be a system of ring polynomial equations with constants in $\mathfrak{U} / \mathfrak{B}$, and which is finitely solvable in $\mathfrak{A} / \mathfrak{B}$. $\Sigma$ can be assumed to be of the form

$$
\Sigma=\left\{\varphi_{1}=0 ; j \in J\right\}
$$

where each $\varphi_{1}$ is a ring polynomial with constants in $\mathfrak{U} / \mathfrak{B}$. Replace each constant from $\mathfrak{A} / \mathfrak{B}$ which appears in $\varphi$, by an arbitrary element from $\mathfrak{A}$ which represents it modulo $\mathcal{B}$. The result is a ring polynomial $\varphi_{j}^{\prime}$ with constants in $R$, and it follows that

$$
\Sigma^{\prime}:=\left\{\varphi^{\prime},=z_{1} ; j \in J\right\} \cup\left\{z_{j} \in \mathfrak{B} ; j \in J\right\} \cup\left\{x_{\imath} \in \mathfrak{A} ; i \in I\right\}
$$

(where $z_{l}, j \in J$, are variables not occurring in. $\Sigma$, and $x_{i}, i \in I$, are all the variables occurring in $\Sigma$ ) is finitely solvable in $R$ : Indeed, for a finite subset of $\Sigma^{\prime}$, choose a solution of the corresponding subset of $\Sigma$, pick representatives of this solution out of the respective cosets and compute for the $z$,'s, which of necessity must lie in $\mathfrak{B}$. Thus $\Sigma$ ' is solvable because $R$ is equationally compact, and any solution taken modulo $\mathfrak{B}$ yields a solution of $\Sigma$ in $\mathfrak{A} / \mathfrak{B}$. The other statement is proved by appropriately modifying the above.

The next result exploits the algebraic compactness of the abelian group underlying an equationally compact ring.

Proposition 1.6. Let $R$ be an equationally compact ring, such that $R^{+}$is a reduced group. Then

$$
R \cong \Pi\left(R_{p} ; p \in \mathbf{P}\right)
$$


where each $R_{p}$ is an algebra over the $p$-adic integers $\mathbf{Z}_{p}^{*}$, complete in the p-adic topology, which is Hausdorff.

Proof. The hypotheses on $R$ together with Definition 0.2 imply that

$$
R^{+} \cong \Pi\left(R_{p} ; p \in \mathbf{P}\right)
$$

where each $R_{p}$ is a $\mathbf{Z}_{p}^{*}$-module with the desired properties. Identify the carrier set $R$ with $\Pi\left(R_{p} ; p \in \mathbf{P}\right)$. If we can show that multiplication in $R$ is componentwise multiplication in $\Pi\left(R_{p} ; p \in \mathbf{P}\right)$, then the given (group) decomposition of $R^{+}$will be also ring direct. So let

$$
\bar{a}_{p}:=\left(a_{p}\right)_{p \in \mathbf{P}} \quad \text { and } \quad \bar{b}_{p}:=\left(b_{p}\right)_{p \in \mathbf{P}}
$$

be arbitrary elements of $\Pi\left(R_{p} ; p \in \mathbf{P}\right)$. Fix $q \in P$, and write $\bar{a}_{p}=$ $a_{q}+\left(a_{p}\right)_{p \neq q}$ and $\bar{b}_{p}=b_{q}+\left(b_{p}\right)_{p \neq q}$ (where $a_{q}:=\left(c_{p}\right)_{p \in \mathbf{P}}$ with $c_{p}=0$ for $p \neq q, c_{q}=a_{q}$, and $\left(a_{p}\right)_{p \neq q}:=\left(d_{p}\right)_{p \in \mathbf{P}}$ with $d_{p}=a_{p}$ for $\left.p \neq q, d_{q}=0\right)$; then compute:

$$
\bar{a}_{p} \cdot \bar{b}_{p}=a_{q} \cdot b_{q}+a_{q} \cdot\left(b_{p}\right)_{p \neq q}+\left(a_{p}\right)_{p \neq q} \cdot b_{q}+\left(a_{p}\right)_{p \neq q} \cdot\left(b_{p}\right)_{p \neq q} .
$$

By Proposition $0.3 R_{p}$ is the largest subgroup $H$ of $R^{+}$with the following property: every element of $H$ is divisible (in $H$ ) by all powers of every prime not equal to $p$. From this and the above decomposition it is clear that $R_{p}$ is the set of all elements which are divisible in $R^{+}$by all powers of every prime not equal to $p$. Moreover, since $R_{p}$ has no nonzero elements divisible by every power of $p$, no nonzero element of $R$ can be divisible in $R^{+}$by every power of every prime. Together, this implies that

(1) $a_{q} \cdot b_{q} \in R_{q}$

(2) $a_{q} \cdot\left(b_{p}\right)_{p \neq q}=\left(a_{p}\right)_{p \neq q} \cdot b_{q}=0$

(3) the $q$ th component of $\left(a_{p}\right)_{p \neq q} \cdot\left(b_{p}\right)_{p \neq q}$ is zero.

Thus we have shown that, if $\bar{a}_{p} \cdot \bar{b}_{p}=\bar{c}_{p}$, then for each prime $q, c_{q}=$ $a_{q} \cdot b_{q}$, i.e., multiplication is componentwise.

It remains only to show that the subrings $R_{p}$ are $\mathbf{Z}_{p}^{*}$-algebras, i.e., that, in addition, $(z \cdot r) \cdot s=z \cdot(r \cdot s)=r \cdot(z \cdot s)$ holds for arbitrary $z \in \mathbf{Z}_{p}^{*}$ and $r, s \in R_{p}$. But $\mathbf{Z}_{p}^{*}$ is just the completion of $\mathbf{Z}$ with respect to the $p$-adic topology on $\mathbf{Z}$, and since $R_{p}$ is a $\mathbf{Z}$-algebra with Hausdorff $p$-adic topology the above identities are obtained by lifting in the standard fashion the same identities which hold down on the $\mathbf{Z}$-algebra $R_{p}$. The proof is complete.

The following consequence of the foregoing is interesting in its own right. 
Proposition 1.7. Let $R$ be an equationally compact ring. Then $R$ is a subring of an equationally compact unital ring if and only if $R^{+}$is a reduced group.

Proof. Note first the elementary fact that a direct product of rings is equationally compact if and only if each factor ring is. Now if $R^{+}$is reduced, then by the last result $R \cong \Pi\left(R_{p} ; p \in \mathbf{P}\right)$ such that for each $p$, $\cap\left(p^{n} R_{p} ; n \in \mathbf{N}\right)=(0)$. Now $R_{p}$ is an equationally compact ring (being a factor of one) and for each $n, p^{n} R_{p}$ is an ideal in $R_{p}$, which, moreover, is expressed by the positive formula $(\exists y)\left(x=p^{n} y\right)$; hence $R_{p} / p^{n} R_{p}$ is an equationally compact ring by Proposition 1.5 . Since it also has positive characteristic it is a subring of an equationally compact unital ring, say $S_{p^{n}}$, by Proposition 0.5. By way of the canonical projections of $R_{p}$ onto $R_{p} / p^{n} R_{p}$ we therefore obtain embeddings

$$
R_{p} \rightarrow \Pi\left(R_{p} / p^{n} R_{p} ; n \in \mathbf{N}\right) \rightarrow \Pi\left(S_{p^{n}} ; n \in \mathbf{N}\right)=: S_{p}
$$

Thus $R_{\mathrm{p}}$ is embeddable in the equationally compact unital ring $S_{p}$. Taking products again, we get

$$
R \cong \Pi\left(R_{p} ; p \in \mathbf{P}\right) \rightarrow \Pi\left(S_{p} ; p \in \mathbf{P}\right)=: S
$$

with $S$ an equationally compact unital ring.

Conversely, suppose $R \subseteq S$, where $S$ is equationally compact and unital. Any divisible subgroup of $R^{+}$must annihilate all of $S$ by Proposition 0.6, i.e., must be (0), since $S$ is unital. Thus $R^{+}$is reduced.

In conclusion we record two Lemmata which will have recurrent applications.

LEMMA 1.8. Let $R$ be a subring of an equationally compact ring $S$, and let $\mathscr{T}$ be a topology for $R$ possessing a subbase of neighborhoods of zero consisting of left ideals of $S$, all of which have finite index in $R$ and are positive in $S$. Then $\mathscr{T}$ is quasi-compact.

Proof. Let $\left\{\mathfrak{U}_{1} ; i \in I\right\}$ be the given subbase. Then the family

$$
\mathscr{F}=\left\{r+\mathfrak{A}_{\imath} ; i \in I, r \in R\right\}
$$

is a subbase of open sets for $\mathscr{T}$. Since each $\mathfrak{A}_{i}$ has finite index in $R, \mathscr{F}$ is also a subbase of closed sets for $\mathscr{T}$. By the Alexander Subbase Theorem $\mathscr{T}$ is quasi-compact if every subset of $\mathscr{F}$ satisfying the finite intersection property has a nonempty intersection. So let 


$$
\mathscr{F}_{0}=\left\{r_{j}+\mathfrak{A}_{i j} ; j \in J\right\}
$$

be such a subset. Setting

$$
\Sigma=\left\{x=r_{j}+x_{j} ; j \in J\right\} \cup\left\{x_{j} \in \mathfrak{A}_{i,} ; j \in J\right\},
$$

it is clear that the finite intersection property for $\mathscr{F}_{0}$ just means that $\Sigma$ is finitely solvable in $S$. Since $S$ is equationally compact, Proposition 1.4 guarantees the solvability of $\Sigma$ in $S$. But the substitute for $x$ in any solution of $\Sigma$ lies in the meet of $\mathscr{F}_{0}$.

LEMMA 1.9. Let $R$ be a ring such that either $R$ has an identitiy or $R^{+}$ is torsion. Let $\mathfrak{U}$ and $\mathfrak{B}$ be left ideals of $R$ of finite index, and let $\mathfrak{B}$ be finitely generated. Then $\mathfrak{U} \cdot \mathfrak{B}$ has finite index.

Proof. Since $R / \mathfrak{B}$ is finite, it suffices to show that $\mathfrak{B} / \mathfrak{A} \cdot \mathfrak{B}$ is finite. Let $b_{1}, \cdots, b_{k}$ generate $\mathfrak{B}$ as a left ideal. If $R^{+}$is torsion, let $m$ be a natural number at least as large as each of the orders of $b_{1}, \cdots, b_{k}$. Otherwise, set $m=1$. It follows that

$$
\begin{aligned}
\mathfrak{B}=\cup\left(R b_{1}+\cdots+R b_{k}+b_{n} ; b_{n}=n(1) b_{1}+\cdots+\right. & n(k) b_{k}, \\
& \left.n \in\{0, \cdots, m-1\}^{\{1, \cdots, k\}}\right) .
\end{aligned}
$$

Further let $R=\cup\left(r_{i}+\mathfrak{A} ; i=1, \cdots, l\right)$ be a finite covering of $R$ by cosets of $\mathfrak{A}$, and let $b \in \mathfrak{B}$ be arbitrary. Then

$$
b=\sum_{j=1}^{k} s_{j} \cdot b_{j}+b_{n}
$$

for appropriate $s_{j} \in R$ and $n \in\{0, \cdots, m-1\}^{\{1, \cdots, k\}}$. For each $j=$ $1, \cdots, k$ there exists $i_{j} \in\{1, \cdots, l\}$ such that $s_{l} \in r_{i_{j}}+\mathfrak{A}$ - say, $s_{j}=r_{i_{j}}+a_{j}$ with $a_{1} \in \mathfrak{A}$. Then

$$
\begin{aligned}
b & =\sum_{j=1}^{k} s_{l} \cdot b_{l}+b_{n}=\sum_{l=1}^{k}\left(r_{i_{j}}+a_{j}\right) \cdot b_{l}+b_{n} \\
& =\sum_{j=1}^{k} r_{i_{j}} \cdot b_{l}+b_{n}+\sum_{j=1}^{k} a_{l} \cdot b_{l} \in \sum_{j=1}^{k} r_{i_{j}} \cdot b_{j}+b_{n}+\mathfrak{A} \cdot \mathfrak{B} .
\end{aligned}
$$

Since there are only a finite number of $r_{i}$ 's, $b_{j}$ 's and $b_{n}$ 's, it is now clear that in $\mathfrak{B}$ there can be only a finite number of cosets modulo $\mathfrak{A} \cdot \mathfrak{B}$.

With these tools in hand we turn our attention to the class of rings satisfying A.C.C. 


\section{The noetherian and torsion cases}

LEMMA 2.1. Let $R$ be an equationally compact noetherian ring, and let $\mathfrak{i}$ be a left ideal of $R$ such that the $R$-module $R / \mathfrak{A}$ is subdirectly irreducible. Then $R /$ II is finite.

Proof. 'Suppose not. Since $R / \mathfrak{N}$ is subdirectly irreducible, there is an element $a \notin ? \mathbb{Y}$ contained in each left ideal properly containing $\mathfrak{A}$. In particular, for any $s \in R \backslash \mathfrak{I}$, the left ideal $\mathfrak{I}+R s$ contains $a$. Choose a set $I$ such that $|I|>|R|$, and let

$$
\Sigma=\left\{a-y_{i j}\left(x_{i}-x_{\jmath}\right)=z_{i j} ; i, j \in I, i \neq j\right\} \cup\left\{z_{i j} \in \mathfrak{A} ; i, j \in I, i \neq j\right\} .
$$

$\Sigma$ is finitely solvable: indeed, in any finite subset of $\Sigma$ replace the occurring $x_{l}$ 's by elements of $R$ which lie pairwise in distinct cosets of $\mathfrak{A}$, and then solve. IT is finitely generated, hence positive (Example 1.2), so Proposition 1.4 says that $\Sigma$ is solvable in $R$. But the cardinality of $I$ forces two distinct $x_{t}$ and $x_{l}$ to assume the same value, implying that $a \in \mathfrak{Q}$. This contradiction completes the proof.

Now let $R$ be an arbitrary unital ring. By Birkhoff's Theorem there are subdirectly irreducible (left) $R$-modules $M_{i}, i \in I$, and an $R$-module embedding

$$
R \rightarrow \Pi\left(M_{i} ; i \in I\right)
$$

which is subdirect. Then the $R$-module $M:=\oplus\left(M_{i} ; i \in I\right)$ is faithful. Indeed, suppose $r \in R$ annihilates $M$. If $\pi_{i}: R \rightarrow M_{t}$ is the natural projection given by the subdirect representation above, then $\pi_{l}(r)=\pi_{i}(r \cdot 1)=r \cdot\left(\pi_{i}(1)\right)=0$, since $\pi_{i}(1) \in M_{i} \subseteq M$. But this holds for each $i \in I$, which forces $r=0$. Now endow $R$ with the $M$-topology $\mathscr{T}$ - for $\mathscr{T}$, a neighborhood base of $r \in R$ is given by the sets

$$
O_{r, m_{1}, \cdots, m_{k}}:=\left\{s \in R ; s m_{\imath}=r m_{\imath}, i=1, \cdots, k\right\}, \quad k \in \mathbf{N}, m_{1}, \cdots, m_{k} \in M .
$$

It is well known that $\mathscr{T}$ is a topology for $R$, and, in our case, $\mathscr{T}$ is Hausdorff since $M$ is faithful. Moreover, a subbase of neighborhoods of zero is given by the family of annihilators $\operatorname{ann}_{R}(m), m \in M$. However, by the nature of $M$ each $\operatorname{ann}_{R}(m)$ is the finite intersection $\cap \operatorname{ann}_{R}\left(m_{j}\right)$, where $m_{j} \in M_{i_{j}}$ and $m=\Sigma m_{j} \in \bigoplus M_{i_{j}}$. Hence the family

$$
\left\{\operatorname{ann}_{R}(m) ; 0 \neq m \in M_{i}, i \in I\right\}
$$

is a subbase of neighborhoods of zero. Now for any $i \in I$, and any nonzero $m \in M_{\imath}$, the $R$-module $R / \operatorname{ann}_{R}(m)$ is isomorphic to 
$R \cdot m$. The latter is a proper submodule of the subdirectly irreducible $R$-module $M_{i}$, hence is itself subdirectly irreducible. Collecting all the pieces, we see that there is a subbase of neighborhoods of zero for the topology $\mathscr{T}$ consisting of left ideals $\mathfrak{A}_{,}, j \in J$, such that $R / \mathfrak{A}_{j}$ is a subdirectly irreducible $R$-module. (This observation is implicit in the proof of Lemma 3 in B. Müller [10].) With its help we obtain:

Proposition 2.2. If $R$ is an equationally compact noetherian ring, then the topology $\mathscr{T}$ (as constructed above) is a compact topology for $R$.

Proof. The above remarks together with Lemma 2.1 yield that $\mathscr{T}$ is a Hausdorff topology of $R$ with a subbase for zero consisting of left ideals each of finite index in $R$. Since in a noetherian ring every left ideal is positive (Example 1.2), Lemma 1.8 (putting $R=S$ there) then settles the matter.

An immediate consequence of Proposition 2.2 and Proposition 0.4 is

COROllary 2.3. A noetherian ring $R$ is equationally compact if and only if the $J(R)$-topology is compact.

In the following two Lemmata it is assumed that $R$ is a ring satisfying A.C.C. and having a torsion group underlying it (which, under A.C.C., is equivalent to $R$ having positive characteristic). Let $\chi(R)=$ $n>0$.

LEMMA 2.4. If $R$ is semisimple and equationally compact, then $R$ is finite.

Proof. By Proposition $0.5 Z_{n} * R=: S$ is an equationally compact noetherian ring. It suffices then to show that $S$ is finite. Now $J(S)$ is a positive ideal so by Proposition $1.5 S / J(S)$ is equationally compact; moreover, $S / J(S)$ is noetherian and semisimple, hence finite by Proposition 0.7. Thus it would suffice to know that $J(S)$ is finite. Suppose not; then by the finiteness of $Z_{n}$ there would be elements $r \neq s \in R, z \in Z_{n}$ with $r+z, s+z$ elements of $J(S)$. But then, remembering that $R$ is an ideal in $S$,

$$
0 \neq r-s=(r+z)-(s+z) \in J(S) \cap R=J(R),
$$

a contradiction.

LEMMA 2.5. If $\mathscr{T}$ is an arbitrary compact topology for $R$, then any left ideal $\mathfrak{A}$ of finite index is open in $\mathscr{T}$. 
Proof. Let $\mathfrak{A}$ be generated by $a_{1}, \cdots, a_{m}$. Then $R a_{1}+\cdots+R a_{m}$ is the image of the compact space $R^{m}$ under the continuous map

$$
\left(x_{1}, \cdots, x_{m}\right) \mapsto x_{1} a_{1}+\cdots+x_{m} a_{m},
$$

hence is compact, hence closed. But then

$$
\mathfrak{A}=\cup\left(R a_{1}+\cdots+R a_{m}+z_{1} a_{1}+\cdots+z_{m} a_{m} ; 0 \leqq z_{i}<n, i=1, \cdots, m\right)
$$

is also closed, hence open because of its finite index.

PROPOSITION 2.6. Let $R$ be an equationally compact ring of positive characteristic $n$ and satisfying A.C.C. Then $R$ is a compact ring, and the $J(R)$-topology is the unique compact topology for $R$.

Proof. $S=Z_{n} * R$ is equationally compact by Proposition 0.5 , and also noetherian. By Corollary 2.3 the $J(S)$-topology is compact. Since $J(R) \subseteq J(S)$ it follows that

$$
\cap\left(J(R)^{n} ; n \in \mathbf{N}\right) \subseteq \cap\left(J(S)^{n} ; n \in \mathbf{N}\right)=(0),
$$

i.e., the $J(R)$-topology is Hausdorff. Now $J(R)$ and all its powers are positive by Example 1.2. Thus $R / J(R)$ is equationally compact (Proposition 1.5), and therefore satisfies the hypotheses of Lemma 2.4. Hence $R / J(R)$ is finite. Calling on Lemma 1.9, a simple induction argument yields that $J(R)^{n}$ has finite index for each $n \in \mathbf{N}$. The $J(R)$-topology thus satisfies the hypotheses of Lemma 1.8 (again putting $R=S$ ) and we conclude that it is a compact topology for $R$. Since it possesses a subbase of neighborhoods for zero consisting of left ideals of finite index, it must be coarser than any other compact topology for $R$ by Lemma 2.5. Since distinct compact topologies are never comparable, the $J(R)$-topology is the unique compact topology for $R$.

3. The main results. From now on $R$ will always denote a ring satisfying A.C.C. The analysis of the $J(R)$-topology by a frontal attack, as was possible in the torsion case, eludes us here. We no longer have Lemma 1.9 at our disposal, nor is there any reason to believe that the powers of the radical are positive ideals, tools which found heavy use in the foregoing. We must take a different tack, by constructing first a more amenable topology for $R$ - later it will reveal itself as just a different description of the $J(R)$-topology.

Proposition 3.1. Let $R$ be equationally compact. Then

$$
R \cong R_{1} \oplus \cdots \oplus R_{n}
$$


where, for each $i=1, \cdots, n, R_{i}$ is a $\mathbf{Z}_{p_{i}}^{*}$-algebra, complete in its Hausdorff $p_{i}$-adic topology.

Proof. Suppose $D \neq(0)$ is a divisible subgroup of $R^{+}$. Then $D$ contains as a subgroup either $\mathbf{Q}^{+}$or a Prüfer group $Z\left(p^{\infty}\right)$ for some $p \in \mathbf{P}$. (See I. Kaplansky [8].) Both of these groups have a proper ascending chain of subgroups; since $R \cdot D=\{0\}$ by Proposition 0.6 , the subgroups in these chains are ideals in $R$, contradicting A.C.C. Hence $R^{+}$ is reduced and therefore by Proposition $1.6 R=\Pi\left(R_{p} ; p \in \mathbf{P}\right)$ with the properties described there. But almost all of these $R_{p}$ 's must be zero, again by A.C.C.

LEMMA 3.2. Let $R$ be equationally compact and subdirectly irreducible. Then $R$ is finite.

Proof. Subdirect irreducibility and Proposition 3.1 imply that $R=$ $R_{p_{1}}$ with the properties stated there. Set $p=p_{1}$. In particular, then, $\cap\left(p^{n} R ; n \in \mathbf{N}\right)=(0)$. Hence by subdirect irreducibility there exists $n \in \mathbf{N}$ with $p^{n} R=(0)$, since the $p^{n} R$ are ideals. I.e., there is an $n \in \mathbf{N}$ such that $\chi(R)=p^{n}>0$. By Proposition 2.6, $R$ is a compact ring in which the unique compact topology is the $J(R)$-topology. In particular, $\cap\left(J(R)^{n} ; n \in \mathbf{N}\right)=(0)$. Again subdirect irreducibility implies $J(R)^{n}=$ $(0)$ for some $n$, that is, the $J(R)$-topology is discrete. Being compact also means that $R$ has to be finite, which was the claim.

Let $R$ be equationally compact. From Proposition 3.1 it follows that

$$
\cap(m R ; m \in \mathbf{N})=(0)
$$

Let $0 \neq r \in R$. Then there is an $m \in \mathbf{N}$ such that $r \notin m R$. Zorn's Lemma yields an ideal, say $\mathfrak{A}_{r}$, which is maximal with respect to the property of (1) being an ideal, (2) not containing $r$, and (3) containing $m R$. Let $\mathscr{T}$ denote the topology on $R$ generated by

$$
\left\{a+\mathfrak{A}_{r} ; a \in R, r \in R \backslash\{0\}\right\}
$$

taken as a subbase for the open sets. Being an ideal topology $\mathscr{T}$ is a topology for $R$, and, moreover, is Hausdorff, since $\cap\left(\mathfrak{A}_{r} ; r \in R \backslash\{0\}\right)=$ (0). The following is easily verified:

Lemma 3.3. Let $R$ be equationally compact. Then for $0 \neq r \in R$, $\mathfrak{U}_{r}$ is a positive ideal, being expressible by the positive formula 


$$
\begin{aligned}
& \left(\exists x_{1}\right) \cdots\left(\exists x_{t+1}\right) \vee\left(x=x_{1} a_{1}+\cdots+x_{t} a_{t}+z_{1} a_{1}+\cdots+z_{t} a_{t}+m x_{t+1} ;\right. \\
& \left.\quad z_{i} \in \mathbf{N}, 0 \leqq z_{i}<m\right)
\end{aligned}
$$

where $a_{1}, \cdots, a_{t}$ generate $\mathfrak{U}_{r}$ as left ideal and $m \in \mathbf{N}$ is such that $\mathfrak{A}, \supseteq m R$.

Lemma 3.4. Let $R$ be equationally compact. Then $\mathscr{T}$ is a compact topology.

Proof. It is clear that $\mathfrak{A}_{r}$ is maximal with respect to the property of being an ideal not containing $r$. Thus $R / \mathfrak{A}$, is subdirectly irreducible, satisfies A.C.C., and is equationally compact since $\mathfrak{A}_{r}$ is positive. Hence $R / \mathfrak{A}$, is finite by Lemma 3.2 , i.e., $\mathfrak{A}$, has finite index. Then Lemma 1.8 applies (taking $R=S$ again), implying that $\mathscr{T}$ is quasi-compact, and hence compact.

We are ready to prove:

THEOREM 3.5. The following are equivalent conditions on a ring $R$ satisfying the ascending chain condition on left ideals:

(i) $R$ is a compact ring.

(ii) $R$ is equationally compact.

(iii) $R$ is a subdirect product of a family of finite subdirectly irreducible rings, closed with respect to the product of the discrete topologies of the factors.

(iv) $R$ is an ideal of an equationally compact noetherian ring.

Proof. (i) $\Leftrightarrow$ (ii) follows from Lemma 3.4.

(iii) $\Rightarrow$ (i): is clear since the product topology is compact.

(iv) $\Rightarrow$ (ii): Ideals are positive in noetherian rings, so Proposition 1.5 applies.

(ii) $\Rightarrow$ (iii): For $R$ we have the topology $\mathscr{T}$ as constructed above. Set $R^{x}=R \backslash\{0\}$. The canonical projections $R \rightarrow R / \mathfrak{A}_{r}, r \in R^{x}$, induce an embedding

$$
R \rightarrow \Pi\left(R / \mathfrak{A}_{r} ; r \in R^{x}\right)=: S
$$

representing $R$ as a subdirect product of the finite subdirectly irreducible rings $R / \mathscr{A}_{r}$. To show is that $R$ is closed in $S$, where $S$ is endowed with the product of the discrete topologies on its factors. Identify $R$ with its image in $S$, and suppose that $\bar{a}:=\left(\bar{a}_{r}\right)_{r \in R^{x}}$, where $\bar{a}_{r}=a_{r}+\mathfrak{A}_{r} \in R / \mathfrak{A}_{r}$, lies in the closure of $R$. This means that every subbasic neighborhood of $\bar{a}$ meets $R$, i.e., that for every finite set $r_{1}, \cdots, r_{n} \in R^{x}$ there exists $b \in R$ such that $b+\mathfrak{A}_{r_{i}}=\bar{a}_{r_{i}}$, i.e., such that $b-a_{r_{i}} \in \mathfrak{A}_{r_{i}}, i=1, \cdots, n$. But that just means that 


$$
\Sigma=\left\{x-a_{r}=y_{r} ; r \in R^{x}\right\} \cup\left\{y_{r} \in \mathfrak{A}_{r} ; r \in R^{x}\right\}
$$

is finitely solvable in $R$. Since the $\mathfrak{A}_{r}$ 's are positive in $R$ (Lemma 3.3) $\Sigma$ is solvable in $R$ by Proposition 1.4, yielding an element $a \in R$ (the substitute for $x$ ) with $a=\left(\bar{a}_{r}\right)_{r \in R^{x}}=\bar{a}$, that is to say, $\bar{a} \in R$.

(ii) $\Rightarrow$ (iv): Let $R=R_{1} \oplus \cdots \oplus R_{n}$ be the decomposition of $R$ given by Proposition 3.1. Since each $R_{i}$ also has A.C.C. and is equationally compact, it suffices to prove the claim for each of the summands; that is, $R$ may be assumed to be a $\mathbf{Z}_{p}^{*}$-algebra and complete in its Hausdorff $p$-adic topology. Set $S=\mathbf{Z}_{p}^{*} * R$. $S$ is noetherian, $R$ is an ideal of $S$, so the claim will be proved if a compact topology for $S$ can be found. Let $\mathscr{T}_{s}$ be the topology on the set $\mathbf{Z}_{p}^{*} \times R$ given by taking the product of the (compact!) $p$-adic topology on $\mathbf{Z}_{p}^{*}$ with the topology $\mathscr{T}$ on $R$, which is compact by Lemma 3.4. Thus $\mathscr{T}_{s}$ is compact, and we claim it is a topology for $S$. Now obviously $\mathscr{T}_{S}$ is compatible with the addition (being componentwise) and it is equally clear that the multiplication map, when restricted to either of the factors $\mathbf{Z}_{p}^{*}$ or $R$, is continuous. Thus the only point of concern is the continuity of the map

$$
\begin{aligned}
\mu: \mathbf{Z}_{p}^{*} \times R & \rightarrow R \\
(z, s) & \mapsto z \cdot s .
\end{aligned}
$$

Now $z \cdot s+\mathfrak{U}_{r}$ is a typical subbasic neighborhood of $z \cdot s$. By construction $\mathfrak{A}_{r}$ contains $m R$ for some $m \in \mathbf{N}$. Then for some $n \in \mathbf{N}, m \mathbf{Z}_{p}^{*}=$ $p^{n} \mathbf{Z}_{p}^{*}=\left(p^{n}\right)$, a neighborhood of zero in the $p$-adic topology on $\mathbf{Z}_{p}^{*}$. Moreover, $\mathfrak{A}_{r}$ is a $\mathbf{Z}_{p}^{*}$-algebra ideal, since every element in $\mathbf{Z}_{p}^{*}$ is the sum of some integer and an element of $\left(p^{n}\right)$ and

$$
\left(p^{n}\right) \cdot \mathfrak{A}_{r}=p^{n} \cdot \mathbf{Z}_{p}^{*} \cdot \mathfrak{A}_{r}=m \cdot \mathbf{Z}_{p}^{*} \cdot \mathfrak{A}_{r} \subseteq m \cdot R \subseteq \mathfrak{A}_{r} .
$$

Thus, $\left(z+\left(p^{n}\right)\right) \times\left(s+\mathfrak{A}_{r}\right)$ is a neighborhood of $(z, s)$ and an easy computation now shows that it is mapped by $\mu$ into $z \cdot s+\mathfrak{A}$. The proof is complete.

REMARK. Implicit in the proof of (ii) $\Rightarrow$ (iv) above is the following: If $R$ is an equationally compact ring satisfying A.C.C., then (in the notation of Proposition 3.1) $R$ is even a compact topological $A$-algebra, where $A=\mathbf{Z}_{p_{1}}^{*} \oplus \cdots \oplus \mathbf{Z}_{p_{n}}^{*}$ is endowed with its (unique) compact topology and $R$ with its compact topology $\mathscr{T}$.

We return now to the radical topology; now, condition (iv) of Theorem 3.5 offers a suitable setting in which to study it.

LEMMA 3.6. If $R$ is equationally compact, then $J(R)^{n}$ has finite index, for every $n \in \mathbf{N}$. 
Proof. Since $J(R)$ is positive, $\bar{R}:=R / J(R)$ is equationally compact by Proposition 1.5, satisfies A.C.C., and is semisimple. Then by Theorem 3.5 (iv) $\bar{R}$ is an ideal of an equationally compact noetherian ring $S$. Then $S / J(S)$ is equationally compact, semisimple and noetherian, hence finite by Proposition 0.7. But $S / J(S)$ is at least as large as $\bar{R} /(J(S) \cap \bar{R})$; since $J(S) \cap \bar{R}=J(\bar{R})=(0)$, we conclude that $\bar{R}$ is finite, i.e., that $J(R)$ has finite index in $R$. Thus, the Lemma will be proved if we show the following: For each $n \geqq 1, J(R)^{n} / J(R)^{n+1}$ is finite.

Now let $S$ be an equationally compact noetherian ring containing $R$ as an ideal (Theorem 3.5 (iv) again). $J(R)$ is then an ideal in $S$, being the meet of $J(S)$ and $R$, two ideals in $S$. Hence every power of $J(R)$ is an ideal in $S$, and therefore positive in $S, S$ being noetherian; hence by Proposition $1.5 J(R)^{n} / J(R)^{n+1}$ is an equationally compact ring. On the other hand, $R / J(R)^{n+1}$ is a left $R$-module, and has, as such, the ascending chain condition on submodules. This means that the $R$-module

$$
M:=J(R)^{n} / J(R)^{n+1}
$$

is finitely generated over $R$; but $J(R) \subseteq \operatorname{ann}_{R}(M)$, and so $M$ is a module over $R / J(R)=\bar{R}$, and finitely generated as such. Since $\bar{R}$ is finite, we conclude that $M$ is even finitely generated as an abelian group. By the Fundamental Theorem, if $M$ were infinite, $M$ would contain a copy of $\mathbf{Z}$ as a (group) direct summand. But the group $M$ is the abelian group underlying the equationally compact ring $J(R)^{n} / J(R)^{n+1}$, hence must be equationally compact as a group, and therefore the summand $\mathbf{Z}$ would be equationally compact as a group too. This is, however, not the case (see e.g. [11]), and therefore $M$ must be finite.

Proposition 3.7. If $R$ is equationally compact, then the $J(R)$ topology is compact.

Proof. By Theorem 3.5 (iv) $R$ is an ideal of an equationally compact noetherian ring $S$. Since the $J(S)$-topology is compact, it follows as before that

$$
\cap\left(J(R)^{n} ; n \in \mathbf{N}\right) \subseteq \cap\left(J(S)^{n} ; n \in \mathbf{N}\right)=(0),
$$

i.e., the $J(R)$-topology is Hausdorff. As observed in the proof of Lemma 3.6, the powers of $J(R)$ are all positive in $S$; this fact and Lemma 3.6 itself mean that the conditions of Lemma 1.8 are met. We conclude that the $J(R)$-topology is compact.

THEOREM 3.8. If $R$ is a compact ring satisfying A.C.C., then the $J(R)$-topology is the unique compact topology for $R$. 
Proof. In the light of Proposition 3.7 it will suffice to show that the topology $\mathscr{T}$ is the coarsest compact topology for $R$. So let $\mathscr{T}_{0}$ be an arbitrary compact topology for $R$. To verify is that each subbasic $\mathscr{T}$-neighborhood of zero, $\mathfrak{A}_{r}$, is $\mathscr{T}_{0}$-open. Let $a_{1}, \cdots, a_{t}$ be left ideal generators of $\mathfrak{A}$, and let $m \in \mathbf{N}$ be such that $\mathfrak{A} \supseteq 2 m R$. Then

$$
A:=R a_{1}+\cdots+R a_{t}+m R
$$

is the image of $R^{t+1}$ (equipped with the $\mathscr{T}_{0}$ power topology) under the continuous map

$$
\left(x_{1}, \cdots, x_{t+1}\right) \mapsto x_{1} a_{1}+\cdots+x_{t} a_{t}+m x_{t+1} ;
$$

hence $A$ is compact, hence $\mathscr{T}_{0}$-closed in $R$. Thus

$$
\mathfrak{A}_{r}=\cup\left(A+z_{1} a_{1}+\cdots+z_{t} a_{t} ; 0 \leqq z_{i}<m, 1 \leqq i \leqq t\right)
$$

is also $\mathscr{T}_{0}$-closed, hence $\mathscr{T}_{0}$-open since $\mathfrak{U}_{r}$ has finite index.

With the help of the above we obtain another characterization of equational compactness. The details missing for the proof are standard ring-topological arguments, which may be supplied by the reader.

THEOREM 3.9. A ring $R$ satisfying A.C.C. is equationally compact if and only if $R / J(R)^{n}$ is finite for each $n \in \mathbf{N}, \cap\left(J(R)^{n} ; n \in \mathbf{N}\right)=(0)$, and $R$ is complete in the $J(R)$-topology.

Note that in the unital or torsion case, the condition that each power of $J(R)$ have finite index in the above can be replaced by the weaker condition that just $R / J(R)$ be finite - on account of Lemma 1.9. However, in the general case the finiteness of $R / J(R)$ will not suffice - the abelian group $\mathbf{Z}$ endowed with the trivial multiplication provides a simple example.

\section{REFERENCES}

1. N. Bourbaki, Élements de mathématique, Livre III, Topologie générale, Chap. III Paris (1951).

2. S. Bulman-Fleming, On equationally compact semilattices, Algebra Universalis, 2 (1972), 146-151.

3. G. Grätzer, Universal Algebra, Van Nostrand, Princeton, N.J. (1968).

4. D. K. Haley, On compact commutative noetherian rings, Math. Annalen, 189 (1970), 272-274.

5. - Equationally compact artinian rings, Canad. J. Math., 25 (1973), 273-283.

6. I. N. Herstein, Noncommutative Rings, Carus Math. monographs, John Wiley \& Sons, Menasha, Wis. (1968). 
7. I. Kaplansky, Topological rings, Amer. J. Math., 69 (1947), 153-183.

8. - Infinite Abelian Groups, University of Michigan Press, Ann Arbor (1954).

9. J. Koś, Abelian groups that are direct summands of every abelian group which contains them as pure subgroups, Fund. Math., 44 (1957), 84-90.

10. B. Müller, On Morita duality, Canad. J. Math., 21 (1969), 1338-1347.

11. J. Mycielski, Some compactifications of general algebras, Colloq. Math., 13 (1964), 1-9.

12. W. Taylor, Atomic compactness and graph theory, Fund. Math., 65 (1969), 139-145.

13. - On equationally compact semigroups, Semigroup Forum, 5 (1972), 81-88.

14. - Pure compactification in quasi-primal varieties, Canad. J. Math., (to appear).

15. R. B. Warfield, Jr., Purity and algebraic compactness for modules, Pacific J. Math., 28 (1969), 699-719.

16. S. Warner, Compact rings and Stone-Čech compactifications, Arch. Math., 11 (1960), 327-332.

17. Compact rings, Math. Annalen, 145 (1962), 52-63.

18. B. Wegłorz, Equationally compact algebras (I), Fund. Math., 59 (1966), 289-298.

19. G. H. Wenzel, Subdirect irreducibility and equational compactness in unary algebras $\langle\boldsymbol{A} ; f\rangle$, Arch. Math., 21 (1970), 256-264.

20. O. Zariski and P. Samuel, Commutative Algebra, Vol. I \& II, Van Nostrand, Princeton, N.J. (1960).

Received August 21, 1975.

UNIVERSITÄT MANNHEIM (WH) 




\section{Pacific Journal of Mathematics}

\section{Vol. 62, No. $1 \quad$ January, 1976}

Mieczyslaw Altman, Contractor directions, directional contractors and

directional contractions for solving equations . .................. 1

Michael Peter Anderson, Subgroups of finite index in profinite groups .........

Zvi Arad, Abelian and nilpotent subgroups of maximal order of groups of odd order

John David Baildon and Ruth Silverman, On starshaped sets and Helly-type theorems ..........................................

John W. Baker and R. C. Lacher, Some mappings which do not admit an

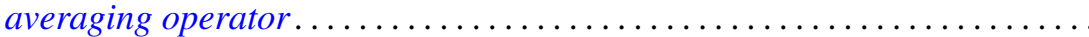

Joseph Barback, Composite numbers and prime regressive isols . . . . . . . . . .

David M. Boyd, Composition operators on $H^{p}(A) \ldots \ldots \ldots \ldots \ldots \ldots \ldots$

Maurice Chacron, Co-radical extension of PI rings . . . . . . . . . . . . .

Fred D. Crary, Some new engulfing theorems . . . . . . . . . . . . . . .

Victor Dannon and Dany Leviatan, A representation theorem for convolution transform with determining function in $L^{p} \ldots \ldots \ldots \ldots \ldots \ldots \ldots \ldots \ldots \ldots \ldots \ldots \ldots \ldots$

Mahlon M. Day, Lumpy subsets in left-amenable locally compact

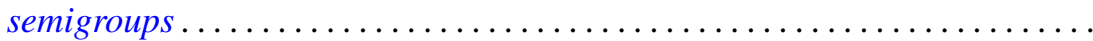

Michael A. Gauger, Some remarks on the center of the universal enveloping algebra of a classical simple Lie algebra . .

David K. Haley, Equational compactness and compact topologies in rings

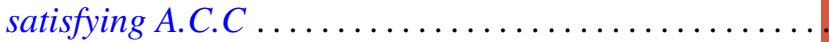

Raymond Heitmann, Generating ideals in Prüfer domains .

Gerald Norman Hile, Entire solutions of linear elliptic equations with

Laplacian principal part. .

Richard Oscar Hill, Moore-Postnikov towers for fibrations in which $\pi_{1}$ (fiber) is non-abelian

John Rast Hubbard, Approximation of compact homogeneous maps . .

Russell L. Merris, Relations among generalized matrix functions . .

V. S. Ramamurthi and Edgar Andrews Rutter, On cotorsion radicals ...

Ralph Tyrrell Rockafellar and Roger Jean-Baptiste Robert Wets, Stochastic

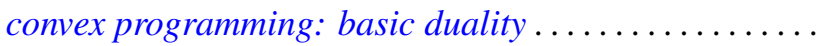

Alban J. Roques, Local evolution systems in general Banach spaces ..

I. Bert Russak, An indirect sufficiency proof for problems with bounded state variables.

Richard Alexander Sanerib, Jr., Ultrafilters and the basis property. .

H. A. Seid, The decomposition of multiplication operators on $L_{p}$-spaces . .

Franklin D. Tall, The density topology .................. 